\title{
Sequential Outage Checkers for Analyzing Cascading Outages and Preventing Large Blackouts
}

\author{
Jin Hur ${ }^{\dagger}$, Manho Joung* and Ross Baldick**
}

\begin{abstract}
This paper presents the use of sequential outage checkers to identify the potential cascading processes that might lead to large blackouts. In order to analyze cascading outages caused by a combination of thermal overloads, low voltages, and under-frequencies following an initial disturbance, sequential outage checkers are proposed. The proposed sequential outage checkers are verified using the AEP 9-bus system, New England 39-bus system, and IEEE 118-bus system.
\end{abstract}

Keywords: Sequential outage checker, Cascading outages, Large blackouts

\section{Introduction}

Large blackouts in power system are generally caused by a consecutive series of outages following an initial disturbance or disturbances. Once a critical component has failed, outages including generator and load trips can sequentially spread and lead to large blackouts. Due to the lack of detailed blackout data and complicated interactions, it is very difficult to a priori identify critical components that weaken the power system and to analyze cascading outages. Cascading outages have been the main cause of large blackouts and they are initiated by various events such as line outages caused by overloading, generator tripping resulting directly from under frequency, and load bus tripping due to abnormal voltage problems [1]. They may also be precipitated by incorrect operation of protection equipment.

A general pattern for blackouts involves sequences of outages occurring successively. During this process, if a critical system component fails, then sequential generator, load, or transmission line tripping can lead to large blackouts [2], [3]. Cascading outage analysis is very important to the understanding and prevention of large blackouts.

Traditionally, electric power systems have been designed and planned to withstand various disturbances, and system reliability has been enhanced by single (N-1) contingency or double (N-2) contingency analysis study. Despite efforts to maintain system reliability, electric power systems are potentially vulnerable to cascading outages in real power system operations [4], [5]. Most steady-state models based on power flow analysis evaluate the consequences for a

$\dagger$ Corresponding Author: Department of Electrical and Computer Engineering, The University of Texas at Austin, USA. (jinhur@mail.utexas.edu)

* Lower Colorado River Authority, USA. (Manho.Joung@1cra.org)

** Department of Electrical and Computer Engineering, The University of Texas at Austin, USA. (baldick@ece.utexas.edu)

Received: December 13, 2010; Accepted: May 19, 2011 given contingency taking into account one possible outage. Such models are not designed for cascading outages that may be triggered by subsequent outage events. Moreover, modeling and analysis of successive combinations of possible outages involves significant computational effort.

Another difficulty with modeling cascading outages is that the process cannot be modeled through steady state analysis alone. That is, dynamic analysis and representation of protection devices is necessary. A typical type of protective scheme among protection devices for generators and loads is the use of under-frequency relays. When the system frequency drops below a threshold value for a pre-specified time period, the protective relay may be triggered and activated [6]. A simulation that combines a steady state model with at least some representation of a dynamic model is therefore needed.

In this paper, sequential outage checkers are proposed to identify potential cascading process that might lead to large blackouts. In order to assess vulnerability to cascading outages due to thermal overloads, under voltages, and low frequency following initial disturbances, three cascading outage checkers, the line outage checker (LOC), the voltage outage checker (VOC) and the frequency outage checker (FOC), are implemented. These outage checkers are operated sequentially to determine the status of the resulting operating state or equilibrium. Some resulting operating states would result in protection equipment removing more elements from the system and therefore potentially precipitating further outages. Among the three checkers, the line outage checker and voltage outage checker use the power flow algorithm, either full AC power flow or decoupled power flow. These outage checkers concentrate on cascading events that can be analyzed by a steady-state model based on power flow calculation. In addition to the steady-state analysis, another outage checker is applied for protection against system over- or under-frequency events.

The rest of this paper is organized as follows. Section 2 
Table 1. Key causes of large blackouts [7]-[12]

\begin{tabular}{c|c|c|c|c|c}
\hline Year & Blackouts & Initial Disturbance & Overload Line & Low Voltage & Low Frequency \\
\hline 1996 & US-Western & $500 \mathrm{k}$ line outage & $\mathrm{Y}$ & $\mathrm{Y}$ \\
2003 & US-Northeastern & $345 \mathrm{k}$ line outage & $\mathrm{Y}$ & $\mathrm{Y}$ \\
2003 & Italy & Interconnecting line outage & $\mathrm{Y}$ & $\mathrm{Y}$ \\
2006 & Europe & line outage & $\mathrm{Y}$ & $\mathrm{N}$ \\
2006 & Japan & line outage & $\mathrm{Y}$ & $\mathrm{N}$ \\
2008 & US-Florida & 138k line swithcing outage & $\mathrm{Y}$ & $\mathrm{Y}$ & \\
\hline
\end{tabular}

introduces patterns of cascading outages from historical blackouts and current approaches to analyze cascading outages. Section 3 describes the operational algorithms which are applied to the proposed sequential outage checkers. Section 4 reports the simulation results of the proposed sequential outage checkers applied to the AEP 9bus test system, the IEEE 39-bus test system, and the IEEE 118-bus test system. Conclusions and future works are given in Section 5.

\section{Cascading Outages Leading To Large Blackouts}

Cascading outages and large blackouts still remain a difficult and complex issue. In this regard, simulation models or analysis techniques for cascading outages can help to prevent large blackouts. Most large blackouts are caused by a sequence of cascading outages. In this section, from the investigation of recent blackouts, the characteristics of sequential events are analyzed and then current approaches to study them will be provided.

\subsection{Characteristics of Cascading Outages}

There have been many cascading outages resulting in large blackouts such as the 1996 US-Western blackout [7], the 2003 US-Northeastern blackout [8], the 2003 Italy blackout [9], the 2006 Europe blackout [10], the 2006 Japan blackout [11], and the 2008 US-Florida blackout [12]. Table 1 summarizes major blackouts with regard to common patterns of cascading outages following the initial disturbances. From the study of blackouts shown in Table 1, line outage as the first outage was typically initiated by protective relay failure, phase-to-ground fault, or a line sagging into a tree [13].

The sequence of cascading outage is well described in [1], [6]. One component outage may create new operating conditions that trigger another outage, which can bring sequential outages such as line tripping, generator tripping, or load shedding. These subsequent outages cause line overloading, low voltage, or under-frequency in the power system. These common patterns provide motivation for implementing cascading outage checkers to analyze cascading outages and to prevent large blackouts.

\subsection{Current Approaches to Analyze Cascading Outages}

Many studies have been performed to identify the sequence of cascading outages that lead to large blackouts. To anticipate the development of cascading outages due to thermal overloads, a cluster approach is proposed [14]. A cluster approach provides a practical process to identify initial disturbances that may result in cascading outages and enables system operators and planners to determine possible cascading chains.

The proposed approach in this paper considers sequential outage analysis based on $\mathrm{AC}$ power flow by checking three kinds of system operating condition: thermal overloads, low voltage, and under-frequency successively. It reveals critical paths that may lead to cascading outages and large blackouts. Because it is very difficult to take into account all contingency cases, it can be more practical to find potential critical paths or possible cascading chains that are common to several initiating events.

It has been recognized that thermal overloads and low voltage are the main cause of cascading outages. In [15], under credible contingency conditions, sequential processes are simulated by monitoring line overloads. After the system status reaches a stable state from the previous simulations, subsequent simulation is carried out for another disturbance until the system fails to coverage to a post-disturbance equilibrium.

In [16], [17], the overload and voltage problems are evaluated by flow contribution factor and voltage contribution factor to predict the possible successive outages at early system state. In the proposed approach, under-frequency contribution factor is considered to represent system dynamics in addition to steady-state analysis representing thermal overloads and low voltage.

\section{Proposed Sequential Outage Checkers}

As mentioned above, key factors to cause cascading outages and large blackouts include system conditions of overloading, low voltage, and under-frequency. This section describes the representation of these issues in a sequential outage checker. 


\subsection{Line Outage Checker (LOC)}

Line overloading for violating thermal limits is an important and common measure to identify the mechanism of cascading outages and to assess vulnerability to cascading outages [16]. In a cascading outage scenario, a line outage can lead to overloading and tripping of other lines.

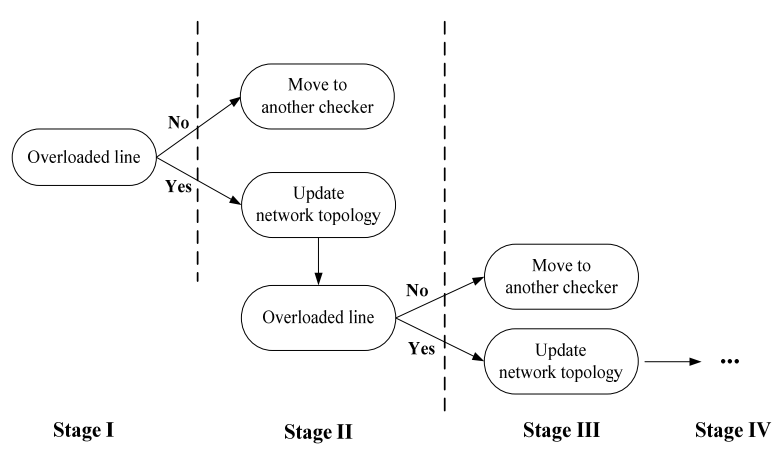

Fig. 1. Operating procedure of the LOC

Fig. 1 shows the concept of the line outage checker (LOC). In Stage I, if an overloaded line is detected, this line is disconnected from the system and the line outage checker is activated again after updating network topology in Stage II. This process will be repeated until no overloaded lines are detected in any stages. If no overload is detected at one stage, a new round of outage checking would be initiated for the next stage.

\subsection{Voltage Outage Checker (VOC)}

Another typical characteristic of cascading outages includes low voltage problems. A steady-state model based on power flow analysis evaluates voltage profiles along with transmission line overloads. After outages, the voltage profiles of power systems may decline. Similarly with a line outage checker, when a voltage profile for each bus violates a pre-defined threshold, the voltage outage checker (VOC) is activated. If a low voltage violates a limit, load shedding action may be taken to maintain bus voltages [18].

\subsection{Frequency Outage Checker (FOC)}

A steady-state analysis model cannot present all possible cascading outage scenarios that lead to large blackouts. In order to analyze cascading outages due to the dynamics of power systems focusing on typical protection devices, a specific model to represent the dynamic behavior of protection device is necessary. If some generators trip, a subsequent frequency excursion will occur, which may trigger the network under-frequency protection. As a result of the frequency excursion, some actions such as load curtailments, load shedding, or voltage reduction can be used to maintain system security.

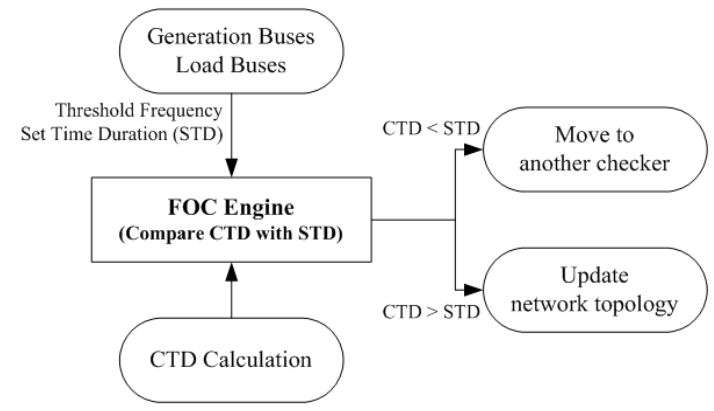

Fig. 2. Operating logic of the FOC

To represent this possibility, the frequency outage checker (FOC) models over- and under-frequency protection. In the frequency outage checker, the system frequency response (SFR) model [19] is used as a frequency change model. The idea of uniform or average frequency is the basic concept in the SFR model, where synchronizing oscillations between generators are filtered out, but the average frequency behavior is retained. The basic SFR model averages the machine dynamic behavior in a large system into an equivalent single machine and it is a representation of only the average system dynamics ignoring the inter-machine oscillations. In this paper, frequency outage is modeled based on the frequency outage standard from ERCOT [20]. According to this standard, when the system frequency is outside an acceptable range for a certain amount of time, an underfrequency trip occurs.

Fig. 2 shows operating procedures of the frequency outage checker. The FOC obtains a pre-defined threshold frequency and time duration information for frequency relays from the input database. The calculated time duration (CTD) block calculates the time duration for a given under or over frequency threshold. In the CTD block, a frequency response function (1) is used to calculate time duration [19]:

$$
f(t)=\frac{R P_{\text {step }}}{\left(D R+K_{m}\right) 2 \pi}\left[1+\alpha e^{-\zeta \omega_{n} t} \sin \left(\omega_{r} t+\phi\right)\right]+60
$$

where,

$$
\begin{aligned}
& \omega_{n}^{2}=\frac{D R+K_{m}}{2 H R T_{R}} \\
& \zeta=\left(\frac{2 H R+\left(D R+K_{m} F_{H}\right) T_{R}}{2\left(D R+K_{m}\right)}\right) \\
& \alpha=\sqrt{\frac{1-2 T_{R} \zeta \omega_{n}+T_{R}^{2} \omega_{n}^{2}}{1-\zeta^{2}}} \\
& \omega_{r}=\omega_{n} \sqrt{1-\zeta^{2}} \\
& \phi=\tan ^{-1}\left(\frac{\omega_{r} T_{R}}{1-\zeta \omega_{n} T_{R}}\right)-\tan ^{-1}\left(\frac{\sqrt{1-\zeta^{2}}}{-\zeta}\right)
\end{aligned}
$$


In the above equations, $R$ is governor droop, $\Delta \omega$ is incremental speed in per unit, $F_{H}$ is fraction of total power generated by the $H P$ turbine, $T_{R}$ is reheat time constant in seconds, $H$ is inertia constant in seconds, $D$ is damping factor, $K_{m}$ is mechanical power gain factor, and $P_{\text {step }}$ is disturbance magnitude in per unit.

Fig. 3 shows how to calculate the time duration from the frequency functions. The time when the frequency first drops under the frequency threshold, $t_{l}$, and the time when the frequency first rises up to the threshold again, $t_{2}$, are determined to calculate the time duration $\left(t_{2}-t_{1}\right)$. To determine the intersection of two functions, Eq. (2) is solved [21]. $K$ is the pre-defined threshold frequency. As a result, time duration for a specific threshold frequency can be obtained.

$$
\frac{R P_{\text {step }}}{\left(D R+K_{m}\right) 2 \pi}\left[1+\alpha e^{-\zeta \omega_{n} t} \sin \left(\omega_{r} t+\phi\right)\right]+60=K
$$

As seen in Fig. 3, the lowest point of frequency value is the first local minimum of the frequency curve. This is due to the fact that the curve is a damped sine wave and the later local minima will be closer to $60 \mathrm{~Hz}$. In an optimization problem like calculating minimum frequency $f$ min, the first order condition to find the local minimizer can be applied. If the derivative of the function at one point is zero, then this point is a local minimizer or maximizer. In this problem, it is necessary to find a $t$ that satisfies the equation $\nabla f(t)=0$ in the first cycle of the curve.

After calculating the time duration in the FOC, it determines whether the calculated time duration (CTD) of frequency violation exceeds the set time duration (STD) of the protective relay. If STD is greater than CTD for all generators, the algorithm would proceed to the next outage checker. If supply is reduced due to a generator trip, the lost power is reapportioned to the other generators by participation factors. If a frequency violation is detected in the calculation step, system topology would be updated and

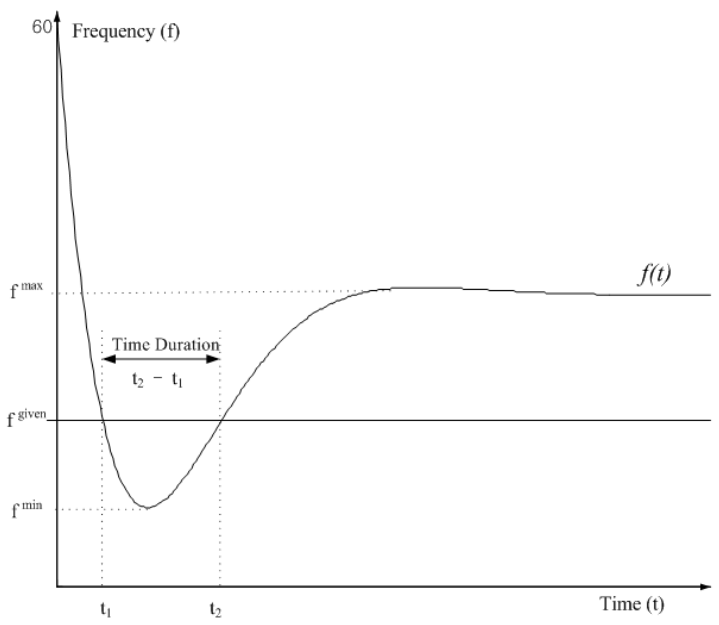

Fig. 3. Time duration $\left(t_{2}-t_{1}\right)$ calculation of the FOC a new round of outage checking would occur in the line overloading or under voltage checker.

\subsection{Algorithm for Sequential Outage Checkers}

Sequential outage checkers test the state of the power system to see if additional outages will be precipitated due to a particular protection criterion. The operational procedures of sequential outage checkers are based on an iterative power flow approach. The algorithm is summarized in Fig. 4.

The algorithm starts with a given initial disturbance and does not consider hidden failures of the protection systems as in [22]. After initiating the disturbance events, cascading outage checkers will be operated in user pre-defined order. For a given disturbance or outage, outage checkers determine the status of the resulting operating state, or equilibrium, and determines if it would result in protection equipment removing more elements from the system and therefore potentially precipitating further outages. If there are several protection actions (or violations) identified by the outage checkers, then timing information from the outage checkers determines which element would be first disconnected. This element is removed from the power flow model using the 'Update topology' blocks. The process then repeats until either a complete system

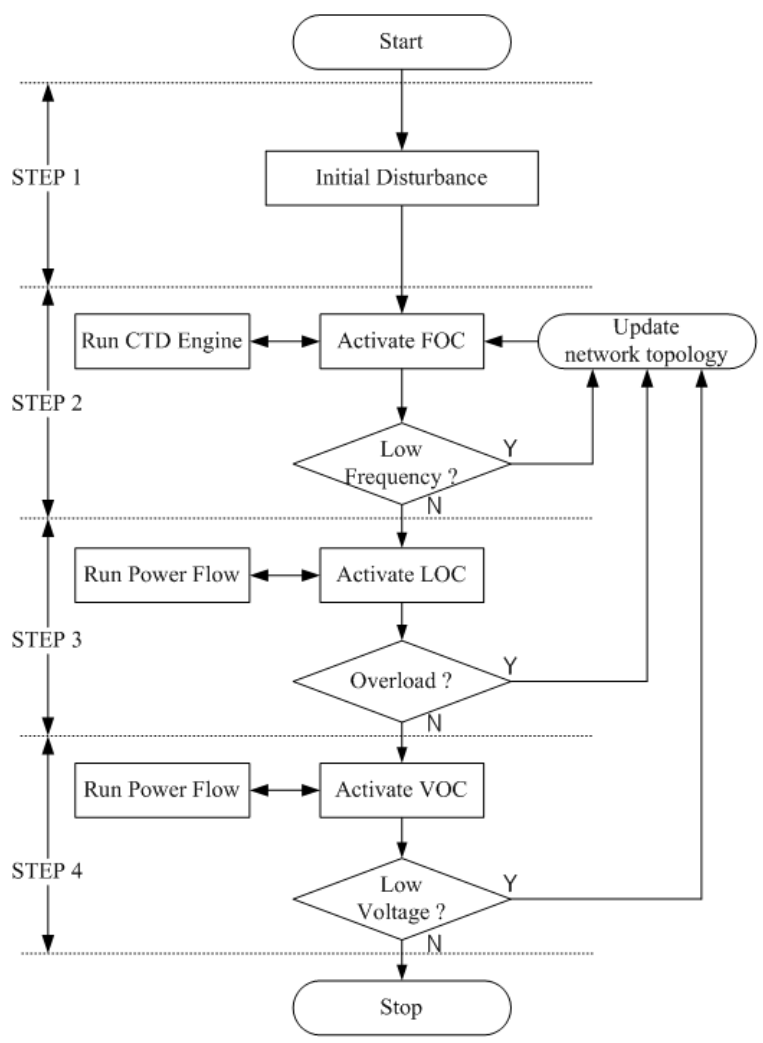

Fig. 4. Operating algorithm of the sequential outage checkers 
blackout occurs (indicated by failure to solve the remaining system) or no more protection actions (no violations) are predicted to occur.

\section{Case Studies}

\subsection{Implementation of Sequential Outage Checkers}

The Cascading outage analysis (COA) tool [21] implementing sequential outage checkers has been built around the Windows-based .NET framework 2.0 in order to support various distributed computing environments. The user interface for the COA tool and the main algorithm of sequential outage checkers has been implemented by visual basic .NET and visual C\#, respectively. After entering input data into the system database, sequential outage checkers are activated according to the initial predefined disturbance. After cascading outage analysis, simulation results are stored in the output database, and are also displayed in tree view form.

In case studies, the proposed sequential outages checkers for analyzing cascading outages and preventing large blackouts are verified by applying to the AEP 9-bus test system, the IEEE 39-bus test system, and the IEEE 118-bus test system. All single line outages as an initial disturbance are considered.

\subsection{AEP 9-Bus Test System}

The AEP 9-bus test system for cascading outage analysis is used for illustration of the proposed sequential outage checkers. In this scenario simulation, cascading outages occur after applying the initial disturbance to the test system. An initial disturbance of tripping line from bus 7 to bus 8 was considered and this initial disturbance caused under-frequency, line overloading, and under voltage events. To describe topology concisely in this and later examples, LA-B will be used to denote a line between A and $\mathrm{B}$. The results of the simulation are as follows:

- Step 1: Line outage of line L7-8

This is the initial disturbance applied to the test system. It is shown in Fig. 5 by the dashed oval around the line. With this line removed, the losses in the rest of the system increase, resulting in a frequency disturbance.

- Step 2: Under-frequency at bus 3

This is the first cascading outage resulting from the initial disturbance.

The information displayed means that the settings of the FOC at the bus 3 are violated causing the opening of the circuit breaker CB1 and the outage of generator 3 . The first cascading outage result is shown in Fig. 5 by the dotted circle around Gen 3. Table 2 shows the set time duration (STD), which is pre-defined by the user; the calculated

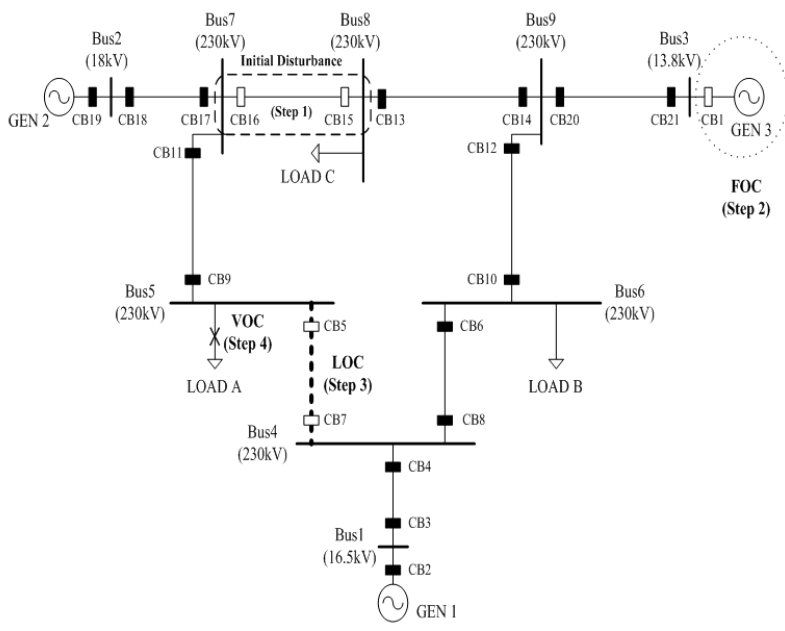

Fig. 5. Cascading outage steps of the AEP 9-bus test system

Table 2. Threshold frequency and STD parameters

\begin{tabular}{c|c|c|c}
\hline Bus & $\begin{array}{c}\text { Threshold } \\
\text { Frequency }(\mathrm{Hz})\end{array}$ & STD (sec) & CTD (sec) \\
\hline 1 & 59.9869 & 6.0 & 2.3071 \\
2 & 59.9869 & 6.0 & 4.5402 \\
3 & 59.9869 & 6.0 & 6.5406 \\
\hline
\end{tabular}

time duration (CTD), which is obtained from the FOC; and threshold frequency. Only bus 3 has a violation detected by the frequency outage checker and CB1 is opened as CTD exceeds STD.

- Step 3: Line overloading from bus 4 and bus 5

This is the second cascading outage resulting from the initial disturbance. The information displayed means that the settings of the line overload checker for the line between buses 4 and 5 are violated causing the opening of circuit breakers $\mathrm{CB} 5$ and $\mathrm{CB} 7$. The second cascading outage is shown in Fig. 5 by the thick dashed line.

- Step 4: Under voltage at bus 5

This is the third cascading outage resulting from the initial disturbance. The information displayed means that the settings of the VOC at bus 5 are violated causing the opening of the circuit breaker CB9 and the loss of load A. The third cascading outage is shown in Fig. 5 by the cross on the distribution feeder to Load A at bus 5 .

The above results are in compliance with the chosen simulation options regarding the order of the outage checkers used. Firstly, the settings of the FOC are checked for violation, then the settings of the LOC and finally the settings of the VOC. Moreover, the FOC whose settings are violated is the one with the highest under frequency threshold, resulting in the outage of generator 3. The tripping of the overload checker at L3-4 is also easy to interpret, since this line has the smallest thermal limit. 
Table 3. Cascading outage results for the IEEE 39-Bus test system

\begin{tabular}{|c|c|c|c|c|c|c|c|c|c|c|c|c|c|}
\hline \multirow{2}{*}{$\begin{array}{c}\text { Initiating } \\
\text { Line Outages }\end{array}$} & \multicolumn{13}{|c|}{ Cascading Outages of the IEEE 39-bus test system } \\
\hline & $1 \mathrm{st}$ & 2nd & $3 \mathrm{rd}$ & 4th & 5 th & 6th & 7 th & 8 th & 9th & 10th & 11 th & 12th & Blackout \\
\hline I6-31 & L9-39 & L10-11 & L2-3 & L1-39 & L29-38 & L10-32 & L22-35 & L19-33 & L20-34 & L23-36 & $\mathrm{L} 2-30$ & $\mathrm{~L} 25-37$ & $\mathrm{~N}$ \\
\hline I10-32 & L6-31 & L9-39 & L1-39 & L29-38 & L22-35 & L19-33 & L2-30 & L23-36 & L20-34 & L25-37 & & & $\mathrm{N}$ \\
\hline I29-38 & L6-31 & L9-39 & & & & & & & & & & & $\mathrm{Y}$ \\
\hline $\mathrm{I} 25-37$ & L16-17 & L15-16 & L6-31 & L9-39 & L1-39 & L29-38 & L2-30 & L10-32 & L16-24 & & & & $\mathrm{N}$ \\
\hline I23-24 & L21-22 & L6-31 & L2-3 & L1-39 & L9-39 & L29-38 & L10-32 & L19-33 & L20-34 & L2-30 & L25-37 & L22-23 & $\mathrm{N}$ \\
\hline I23-36 & L22-23 & L16-24 & & & & & & & & & & & $\mathrm{N}$ \\
\hline I $28-29$ & L26-29 & L16-17 & L15-16 & L6-31 & L9-39 & L1-39 & L10-32 & $\mathrm{L} 2-30$ & L25-37 & L16-24 & & & $\mathrm{N}$ \\
\hline $\mathrm{I} 2-3$ & L26-27 & L1-2 & L6-31 & L10-32 & L9-39 & L22-35 & L19-33 & L20-34 & L23-36 & L25-26 & & & $\mathrm{N}$ \\
\hline I $21-22$ & L23-24 & L6-31 & L2-3 & L1-39 & L9-39 & L29-38 & L10-32 & L19-33 & L20-34 & L2-30 & L25-37 & L22-23 & $\mathrm{N}$ \\
\hline I15-16 & L16-17 & L14-15 & L16-24 & L3-18 & L17-27 & & & & & & & & $\mathrm{N}$ \\
\hline I19-33 & L14-15 & L6-31 & L9-39 & L2-3 & & & & & & & & & $\mathrm{Y}$ \\
\hline $\mathrm{I} 22-35$ & L6-31 & L9-39 & L2-3 & L1-39 & L29-38 & L10-32 & L19-33 & L20-34 & L23-36 & L2-30 & L25-37 & & $\mathrm{N}$ \\
\hline I20-34 & L19-20 & L16-24 & & & & & & & & & & & $\mathrm{N}$ \\
\hline I16-19 & L14-15 & L3-4 & L9-39 & L1-39 & L26-27 & L2-3 & L22-35 & L23-36 & L25-26 & VOC (Bus 8) & & & $\mathrm{N}$ \\
\hline
\end{tabular}

Finally, the outage of L3-4 causes excessive power flow on L5-7 for the supply of load A, which results in the undervoltage settings at bus 5 to be violated. The processes of cascading outages have been identified by the sequential outage checkers.

\subsection{New England 39-Bus Test System}

The New England 39-bus test system is shown in Fig. 6. Fourteen line outages that can cause cascading outages are determined by a single line contingency analysis.

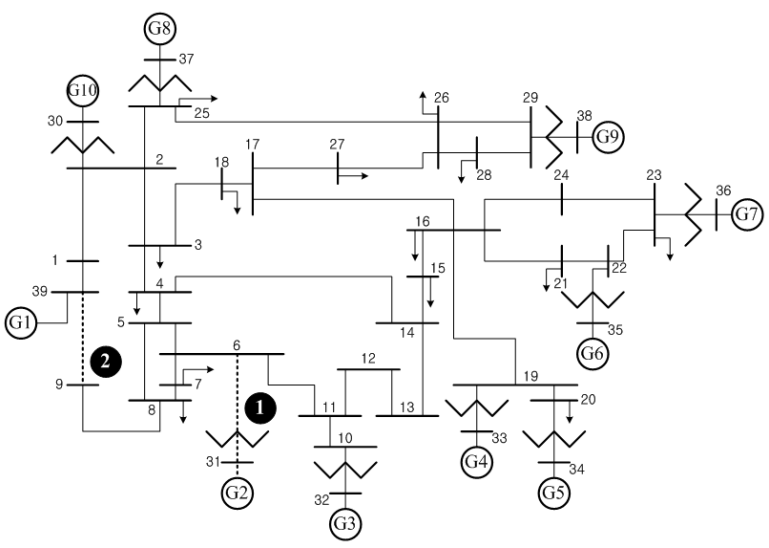

Fig. 6. Critical path of the new England IEEE 39-bus test system

Table 3 shows the cascading outage results for the IEEE 39-bus test system. In Table 3, IA-B denotes the initiating line outage between buses A and B. For example, in Table 3 , when a line outage between buses 19 and 33 (I19-33) occurs, overloaded lines L14-15, L6-31, L9-39, and L2-3 are detected sequentially result in blackout. After initiating a line outage between buses 29 and 38 (I29-38), overloaded lines L6-31 and L9-39 are detected, also resulting in blackout. Among the selected line outages only
I29-38 and I19-33 result in blackouts, as denoted by the last column of Table 3 . They can be regarded as critical lines.

In the case of I16-19, by checking the LOC and VOC, it was discovered that 9 overloading events occurred sequentially and an under voltage occurred at bus 8 at the last step. The VOC used an allowable voltage range from 0.9 to $1.1 \mathrm{pu}$. As shown in Table 3, the critical path L6$31 \rightarrow$ L9-39 shown in Fig. 6 results in a blackout and is common to the sequences following 6 initiating events. General steady state analysis, based on $\mathrm{N}-1$ secure contingency analysis to find critical components, focuses on evaluating only the current status following outages. On the other hand, sequential outage checkers provide the analysis of the sequential multiple system state following the initial disturbance.

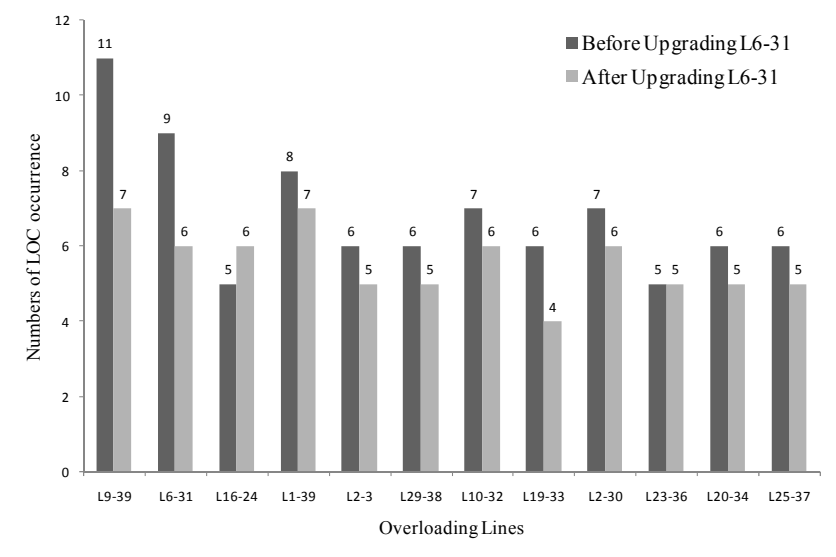

Fig. 7. Effect on system stress levels after upgrading L6-31

Although only the I29-38 and I19-33 initiating events evolve into a blackout, sequential outage analysis can provide important information on system stress related to various initiating events. To alleviate system stress due to overloading lines and to prevent large blackouts, upgrading of lines can be considered. For example, suppose that the 
line thermal capacity of L6-31 is upgraded from 1213.6 to 1577.6 MVA. The reason for selecting L6-31 is that L6-31 is the first step in the critical path subsequent to I10-32, I29-38, and I22-35 initiating events. By upgrading this line, system stress for line overloading in cascading outages and some line outages leading to blackouts are expected to be reduced. Based on the simulation results of the IEEE 39bus test system, the advantages of sequential outage checkers include being able to identify the critical lines and to show the sequential process of cascading outages resulting in blackouts.

\subsection{IEEE 118-Bus Test System}

To verify the proposed sequential outage checkers for a larger system, a numerical test on the IEEE 118-bus test system comprising 54 generation units, 91 loads, and 186 lines is provided in this section. A single line outage for all transmission lines is considered and 23 line outages as an initial disturbance are selected to present cascading outage analysis. Table 4 shows the cascading outage results for the IEEE 118-bus test system. All of the selected line outages displayed in Table 4 results in blackouts following cascading outages. I110-112 and I76-77 line outages reached infeasible solution of power flow calculations following $16^{\text {th }}$ and $17^{\text {th }}$ steps, respectively. On the other hand, I63-64 and I64-65 line outages reached infeasible solution status in much fewer steps. As shown in Table 4, the critical path, L8-30 $\rightarrow$ L5$6 \rightarrow$ L11-12 $\rightarrow$ L3-5 $\rightarrow$ FOC (Bus 12) $\rightarrow$ L11-13 $\rightarrow$ L65-68, leading to blackout is observed among 11 sequences of line outages. As a disturbance power caused by consecutive line outages exceeds $1.0 \mathrm{pu}$ on a 100 MVA base, the FOC would be activated at Bus 12 .

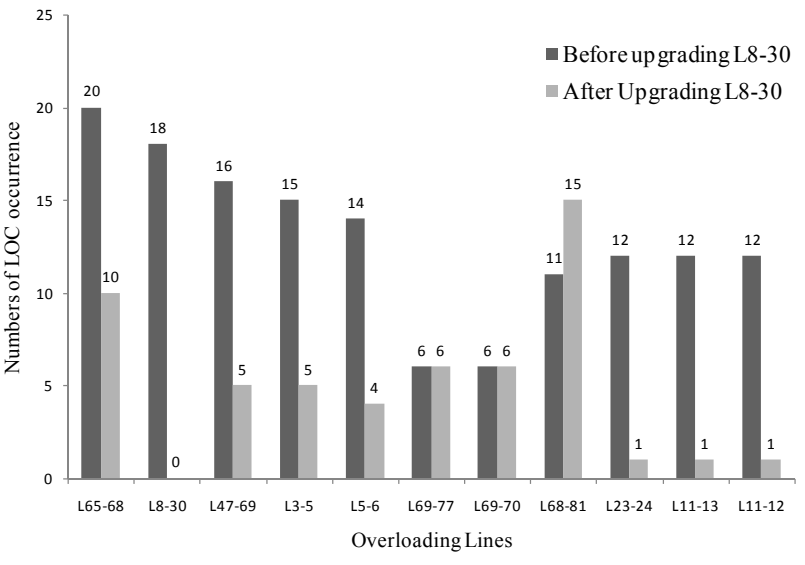

Fig. 8. Effect on system stress levels after upgrading L8-30

Fig. 8 presents the numbers of the LOC occurrence for each line outage in the cascading outages of the IEEE 118bus test system. As shown in Fig. 8, 11 particular lines occurred in sequences of line outages 12 or more times; L65-68 and L8-30 were the most frequent overloading lines. As the analysis using sequential outage checkers provides consecutive outage steps, critical path, and system stress for line overloading leading to blackout, power system reliability can be enhanced by modifying the existing transmission lines by adding or replacing lines. For example, suppose the line thermal capacity of L8-30 is upgraded from 100 to 120 MVA. The reason for selecting L8-30 is that L8-30 is the first step in the critical path and caused the initial cascading outages in the first stage in I89, I9-10, I5-6, I4-11, I5-11, I94-96 and I103-110 line outages. Upgrading this line reduces the system stress for line overloading in cascading outages and can be expected

Table 4. Cascading outage results for the IEEE 118-Bus test system

\begin{tabular}{|c|c|c|c|c|c|c|c|c|c|c|c|c|c|c|c|c|}
\hline \multirow{2}{*}{$\begin{array}{c}\text { Line } \\
\text { Outages }\end{array}$} & \multicolumn{16}{|c|}{ Cascading Outages of the IEEE 118 bus-test system } \\
\hline & 1 st & 2nd & $3 \mathrm{rd}$ & 4th & 5 th & 6th & 7 th & 8 th & 9th & 10th & 11th & 12th & $13 \mathrm{rd}$ & 14th & 15th & 16th \\
\hline I8-9 & L8-30 & L38-65 & L70-71 & L45-46 & L65-68 & L69-70 & L69-77 & L68-81 & L45-49 & & & & & & & \\
\hline $19-10$ & L8-30 & L38-65 & L70-71 & L45-46 & L65-68 & L69-70 & L69-75 & L69-77 & L68-81 & L45-49 & & & & & & \\
\hline $\mathrm{I} 22-23$ & L23-32 & L8-30 & L5-6 & L11-12 & L3-5 & FOC & L11-13 & L65-68 & L71-73 & L69-70 & L69-75 & L68-81 & L69-77 & & & \\
\hline I75-118 & L76-77 & L68-81 & L79-80 & L77-78 & L82-83 & L8-30 & L5-6 & L11-12 & L3-5 & FOC & L11-13 & L65-68 & L69-70 & L69-77 & L69-75 & \\
\hline I76-77 & L75-118 & L68-81 & L79-80 & L77-78 & L82-83 & L8-30 & L5-6 & L11-12 & L3-5 & FOC & L11-13 & L65-68 & L71-73 & L69-70 & L69-77 & L69-75 \\
\hline I79-80 & L77-78 & L68-81 & L82-83 & L8-30 & L5-6 & L11-12 & L3-5 & FOC & L11-13 & L65-68 & L69-70 & L69-77 & L69-75 & & & \\
\hline I4-5 & L5-11 & L5-6 & L3-5 & L8-30 & L38-65 & L70-71 & L45-46 & L65-68 & L69-70 & L69-75 & L69-77 & L68-81 & L45-49 & & & \\
\hline I100-103 & L100-104 & & & & & & & & & & & & & & & \\
\hline I5-6 & L8-30 & L11-12 & L3-5 & L11-13 & L65-68 & L47-69 & L23-24 & & & & & & & & & \\
\hline I4-11 & L8-30 & L5-11 & L5-6 & L3-5 & L65-68 & L47-69 & L71-73 & L69-70 & L68-116 & L69-77 & L $74-75$ & & & & & \\
\hline I5-11 & L8-30 & L5-6 & L4-11 & L3-5 & L65-68 & L47-69 & L71-73 & L69-70 & L69-77 & L74-75 & & & & & & \\
\hline I34-37 & L35-37 & L17-18 & L15-19 & L65-68 & L69-70 & L74-75 & L69-75 & L69-L77 & L68-81 & L38-65 & L45-46 & L45-49 & & & & \\
\hline I85-89 & L85-88 & L79-80 & L69-77 & L82-96 & L77-78 & L68-81 & L8-30 & L5-6 & L11-12 & L3-5 & FOC & L11-13 & L65-68 & L69-70 & L69-75 & \\
\hline $125-27$ & L23-32 & L17-31 & L17-113 & L65-68 & L23-24 & L31-32 & L29-31 & L8-30 & L22-23 & L26-30 & L47-69 & & & & & \\
\hline I63-64 & L59-61 & L59-60 & L49-51 & L56-57 & & & & & & & & & & & & \\
\hline I64-65 & L66-67 & L62-66 & L49-51 & L56-57 & & & & & & & & & & & & \\
\hline I26-30 & L23-32 & L8-30 & L22-23 & L65-68 & L47-69 & L25-27 & & & & & & & & & & \\
\hline I8-30 & L5-6 & L11-12 & L3-5 & FOC & L11-13 & L65-68 & L69-70 & L69-77 & L68-81 & L69-75 & & & & & & \\
\hline I110-112 & L68-81 & L79-80 & L82-83 & L82-96 & L77-78 & L8-30 & L5-6 & L11-12 & L3-5 & FOC & L11-13 & L65-68 & L69-70 & L69-77 & L69-75 & \\
\hline I77-78 & L79-80 & L68-81 & L82-83 & L8-30 & L5-6 & L11-12 & L3-5 & FOC & L11-13 & L65-68 & L69-70 & L69-75 & & & & \\
\hline I94-96 & L8-30 & L5-6 & L11-12 & L3-5 & FOC & L11-13 & L65-68 & L69-70 & L69-77 & L68-81 & L69-75 & & & & & \\
\hline I75-118 & L76-77 & L68-81 & L79-80 & L77-78 & L82-83 & L8-30 & L5-6 & L11-12 & L3-5 & L11-13 & L65-68 & L47-69 & L23-24 & & & \\
\hline I103-110 & L8-30 & L5-6 & L11-12 & L3-5 & FOC & L11-13 & L65-68 & L69-70 & L69-77 & L68-81 & L69-75 & & & & & \\
\hline
\end{tabular}


Table 5. Cascading outage results for the IEEE 118-Bus test system upgrading line 8-30

\begin{tabular}{|c|c|c|c|c|c|c|c|c|c|c|c|c|c|}
\hline \multirow{2}{*}{$\begin{array}{c}\text { Line } \\
\text { Outages }\end{array}$} & \multicolumn{13}{|c|}{ Cascading Outages of IEEE 118 bus-test system after upgrading L8-30 } \\
\hline & $1 \mathrm{st}$ & 2nd & $3 \mathrm{rd}$ & 4th & 5 th & 6th & 7th & 8 th & 9th & 10th & 11th & 12th & Blackouts \\
\hline I8-9 & L65-68 & L65-68 & L47-69 & L71-73 & L69-70 & L68-116 & L74-75 & & & & & & $\mathrm{Y}$ \\
\hline $19-10$ & L65-68 & L47-69 & L71-73 & L69-70 & L68-116 & L74-75 & L69-75 & L69-77 & L68-81 & L45-49 & & & $\mathrm{Y}$ \\
\hline I $22-23$ & L23-32 & L82-83 & L79-80 & L68-81 & L77-78 & L82-96 & & & & & & & $\mathrm{N}$ \\
\hline I75-118 & L75-118 & L68-81 & L79-80 & L77-78 & L82-83 & L82-96 & & & & & & & $\mathrm{Y}$ \\
\hline I76-77 & L75-118 & L68-81 & L79-80 & L77-78 & L82-83 & L82-96 & & & & & & & $\mathrm{N}$ \\
\hline I79-80 & L77-80 & L68-81 & L82-83 & L82-96 & & & & & & & & & $\mathrm{Y}$ \\
\hline I4-5 & L5-11 & L5-6 & L3-5 & L16-17 & & & & & & & & & Y \\
\hline I100-103 & L100-104 & & & & & & & & & & & & Y \\
\hline I5-6 & L5-11 & L4-11 & L3-5 & L16-17 & L65-68 & L69-70 & L69-75 & L68-81 & L69-77 & & & & $\mathrm{Y}$ \\
\hline I4-11 & L5-11 & L5-6 & L3-5 & L16-17 & L65-68 & L69-70 & L69-75 & L68-81 & L69-77 & & & & $\mathrm{Y}$ \\
\hline I5-11 & L5-6 & L4-11 & L3-5 & L16-17 & L65-68 & L69-70 & L69-75 & L68-81 & L69-77 & & & & $\mathrm{Y}$ \\
\hline I34-37 & L35-37 & L17-18 & L15-19 & L65-68 & L69-70 & L74-75 & L69-75 & L69-77 & L68-81 & L38-65 & L45-46 & L45-49 & $\mathrm{Y}$ \\
\hline I85-89 & L85-88 & L79-80 & L69-77 & L82-96 & L77-78 & L68-81 & & & & & & & $\mathrm{N}$ \\
\hline $\mathrm{I} 25-27$ & L23-32 & L17-31 & L17-113 & L65-68 & L23-24 & L31-32 & L29-31 & L22-23 & L26-30 & L47-69 & & & $\mathrm{Y}$ \\
\hline I63-64 & L59-61 & L59-60 & L49-51 & L56-57 & & & & & & & & & Y \\
\hline I64-65 & L66-67 & L62-66 & L49-51 & L56-57 & & & & & & & & & Y \\
\hline I26-30 & L23-32 & L22-23 & L65-68 & L47-69 & L25-27 & & & & & & & & Y \\
\hline I8-30 & L5-6 & L11-12 & L3-5 & L11-13 & L65-68 & L47-69 & L24-24 & & & & & & Y \\
\hline I1 10-112 & L68-81 & L79-80 & L82-83 & L82-96 & L77-78 & & & & & & & & $\mathrm{N}$ \\
\hline I77-78 & L79-80 & L68-81 & L82-83 & L82-96 & & & & & & & & & $\mathrm{N}$ \\
\hline I94-96 & L82-83 & L79-80 & L77-78 & L68-81 & L82-96 & & & & & & & & $\mathrm{N}$ \\
\hline I75-118 & L75-118 & L68-81 & L79-80 & L77-78 & L82-83 & L82-96 & & & & & & & $\mathrm{N}$ \\
\hline I103-110 & L82-83 & L79-80 & L77-78 & L68-81 & L82-96 & & & & & & & & $\mathrm{N}$ \\
\hline
\end{tabular}

to reduce the incidence of blackouts.

Table 5 shows the cascading outages results for the IEEE 118-bus test system after upgrading L8-30. Compared with Table 5 , the number of stages is significantly reduced for most line outages, and 8 line outages such as I22-23, I7677, I85-89, and I110-112, I77-78, I94-96, I75-118 and I103-110 are excluded from the candidate lines leading to blackouts. Additionally, Fig. 8 shows the comparison results of system stress for line overloading after updating L8-30. As shown in Fig. 8, most of the selected overloading lines are alleviated, and no overloading event occurred in L8-30.

On the other hand, even if L65-68 is upgraded to 500 MVA, no line outages are excluded from the candidate lines leading to blackouts, and there is no effect on system stress levels for overloading. As a result, the most important criteria in selecting lines to alleviate system stress and to prevent blackout is to take into account the cascading outage steps and the critical path leading to large blackout as predicted by sequential outage checkers.

\section{Conclusion and Future Work}

Most large blackouts have a common pattern where sequences of outages have occurred successively and have gradually weakened power systems. Main reasons for cascading outages leading to large blackouts are line overloading, low voltage, or under-frequency problems.
Sequential outage checkers to identify successive processes of system outage events leading to large blackouts are needed. In this paper, sequential outage checkers for analyzing cascading outages and preventing large blackouts are presented. To analyze cascading outages, the LOC, the VOC and the FOC are integrated into the cascading outage analysis tool. From the case studies, three key pieces of information for analyzing cascading outages can be provided by sequential outage checkers. Firstly, sequential outage checkers provide the progressive steps of consecutive outage events. This information can enable system operators to understand how an initial disturbance propagates sequentially for a given disturbance. Additionally, this will act as a key to identifying a chain of successive outages, which can result in large blackouts. Secondly, sequential outage checkers suggest a critical path leading to large blackouts. This information can enable system operators to forecast critical operating constraints of transmission flows and help system planners to avoid the potential cascading process that might lead to large blackouts. Finally, the result of sequential outage analysis can inform system operators of system stress levels of transmission overloading and provide the candidate lines to upgrade in order to prevent large blackouts. Consequently, sequential outage checkers will act as efficient tools to analyze cascading outages and to prevent large blackouts.

In our model systems such as the AEP 9-bus test system, New England 39-bus test system, and IEEE 118-bus system, we assumed that the type of all generating units is reheat steam turbine and we used the typical parameters of 
reheat steam turbine units. We calibrated the overall SFR to an effective time constant that is attempting to approximately represent overall time constant. For more general systems, we have to come up with an effective time constant that approximately matches the overall behavior of various types of turbines. In future work, we will develop detailed models with multiple types of turbines representing the fraction of each type of generations.

In modern power systems, various turbine types of generating units would be considered when we study the frequency response analysis. The proposed frequency outage checker can be used for them, but it depends on empirical data from the test system. Empirically, second order model matches the overall behavior of frequency fairly well. Time constants of the second order model may not be same with what we assumed from the reheat steam turbines, but it turns out that the second order model pretty well reflects the characteristics of the behavior [23]. For example, the behavior is pretty much like behavior of second order model even though gas turbine and gas combine cycle generating units are marginal most of the time in ERCOT. So long as this model can be calibrated to the actual behavior of system, the second order model should be sufficient.

To apply sequential outage checkers to a real power system, various types of the initial disturbances causing to the progression to cascading outages should be specified including hidden failures. To accomplish this work, the extension to other types of outages will be implemented into the COA tool, including not only a single line outage but also multiple line outages, generator outages, and substation outages. For practical application of the FOC, the methodologies for determining dynamic system parameters and data acquisition of a protective relay setting will be studied.

\section{References}

[1] K. Yamashita, J. Li, P. Zhang, and C. C. Liu, "Analysis and control of major blackout events" in Proc. IEEE Power Eng. Soc. Power Systems Conf. Expo, pp. 1-4, March 2009.

[2] C. C. Liu and J. Li, "Patterns of cascaded events in blackouts," in Proc. IEEE PES General Meeting, pp. $1-2$, July 2008.

[3] P. Hines, J. Apt, and S. Talukdar, "Trends in the history of large blackouts in the united states," in Proc. IEEE PES General Meeting, pp. 1-8, July 2008.

[4] S. Lee, "Probabilistic reliability assessment for transmission planning and operation including cascading outages," in Proc. IEEE PES Conf. Expo., pp. 1-8, March 2009.

[5] S. Miller, "Extending traditional planning methods to evaluate the potential for cascading failures in electric power grids," in Proc. IEEE PES General
Meeting, pp. 1-7, July 2008.

[6] R. Baldick, B. Chowdhury, et al. "Vulnerability assessment for cascading failures in electric power systems," in Proc. IEEE PES Conf. Expo., pp. 1-9, March 2009.

[7] D. Kosterev, C. Taylor, and W. Mittelstadt, "Model validation for the august 10, 1996 wscc system outage," IEEE Trans. Power Syst., vol. 14, no. 3, pp. 967-979, Aug 1999.

[8] R. Leelaruji and V. Knazkins, "Modeling adequacy for cascading failure analysis," in Proc. Australasian Univ. Power Eng. Conf., pp. 1-6, Dec. 2008,

[9] M. Sforna and M. Delfanti, "Overview of the events and causes of the 2003 Italian blackout," in Proc. IEEE PES Conf. Expo., pp. 301-308, Nov. 2006.

[10] J. De La Ree, Y. Liu, L. Mili, A. Phadke, and L. DaSilva, "Catastrophic failures in power systems: Causes, analyses, and countermeasures," Proc. of the IEEE, vol. 93, no. 5, pp. 956-964, May 2005.

[11] T. Ohno and S. Imai, "The 1987 tokyo blackout," in Proc. IEEE Power Eng. Soc. Power Systems Conf. Expo., pp. 314-318, Nov. 2006.

[12] FRCC System Disturbance and Underfrequency Load Shedding Event Report. FRCC Event Analysis Team, Final Report, 2008.

[13] B. Gou, and H. Zheng, et al. "The statistical law of power system blackouts," in Proc. Annual North American Power Symposium, pp. 495-501, Sept. 2006,

[14] N. Bhatt, S. Sarawgi, et al. "Assessing vulnerability to cascading outages," in Proc. IEEE Power Eng. Soc. Power Systems Conf. Expo., March 2009, pp. 1-9.

[15] B. Chowdhury and S. Baravc, "Creating cascading failure scenarios in interconnected power systems," in Proc. IEEE PES General Meeting, 2006, pp. 1-8.

[16] L. Jinling, C. Yuan, and Z. Yongli, "Identification of cascading failures based on overlaod character of transmission lines," in Proc. Int. Conf. Elec. Utility Deregulation and Restructuring and Power Technologies, pp. 1030-1033, 2008,

[17] N. Zhang, H. Song, and M. Kezunovic, "New monitoring and control scheme for preventing cascading outage," in Proc. Annual North American Power Symposium, pp. 37-42, Oct. 2005.

[18] H. Song and M. Kezunovic, "A new analysis method for early detection and prevention of cascading events," Elect. Power Syst. Res., vol. 77, no. 8, pp. 1132-1142, 2007.

[19] P. Anderson and M. Mirheydar, "A low-order system frequency response model,” IEEE Trans. Power Syst., vol. 5, no. 3, pp. 720-729, Aug. 1990.

[20] ERCOT OPERATING GUIDES: System Operations. ERCOT control area authority operation, 2008.

[21] R. Baldick, J. Hur, et al.’Reducing the Vulnerability of Electric Power Grids to Terrorist Attacks", Department of Energy (DOE), 2009, Final Project 
Report (DOE/ER/25671-1).

[22] L. Mili, and Q. Qiu, et al, "Risk assessment of catastrophic failures in electric power systems," Int. J. of Critical Infrastructures, vol. 1, no. 1, pp. 38-63, 2004.

[23] M. Grady, "Texas Synchrophasor Network Observation Reports (February 2009 through January 11, 2011)", The University of Texas at Austin, January 2011

Jin Hur He received his B.S. and M.S. degree in Electrical Engineering from Korea University, Seoul, South Korea, in 1997 and 1999, respectively. He was a researcher at Korea Electro-technology Research Institute (KERI) from 1999 to 2006. Since 2006, he has been pursuing a Ph.D. degree in the Department of Electrical and Computer Engineering at The University of Texas at Austin.

Manho Joung He received his B.S. and M.S. degree in Electrical Engineering from Seoul National University, Seoul, South Korea, in 1995 and 1997, respectively, and the M.S. degree in Economics and the Ph.D. degree in Electrical and Computer Engineering from the University of Texas at Austin, in 2002 and 2008, respectively. He is currently works as Strategic Energy Analyst, Sr. at Lower Colorado River Authority.

Ross Baldick He received his B.Sc. in Mathematics and Physics and B.E. in Electrical Engineering from the University of Sydney, Australia and his M.S. and Ph.D. in Electrical Engineering and Computer Sciences in 1988 and 1990, respectively, from the University of California, Berkeley. He is currently a Professor in the Department of Electrical and Computer Engineering at The University of Texas at Austin. 